\title{
DIE MÄNNER UND DIE MODERNE
}

\author{
Albrecht Koschorke
}

Around 1900, the term ,modernity" is mainly discussed in categories of gender. The contemporaries consider the cultural development of the $19^{\text {th }}$ century, ending in the epoch of decadence, to be a growing effemination on different levels of collective and individual experience. In contrast to this, the partisans of naturalism have already begun to strike up a tone of pithy virile innovation - a tone iaken over by the manifests of the early avantgarde. Yet they don't relate to real conflicts between the sexes. Instead, the rhetoric of masculinity aims at mastering a specifically modern crisis of differentiation by means of a new culture of will-power and strength of purpose.

Die Moderne-Diskussion um 1900 wird großenteils in Geschlechterkategorien gefuihrt. Während die Entwicklung des 19. Jahrhunderts, die in der décadence einmündet, sich den Zeitgenossen auf unterschiedlichen Ebenen der kollektiven und Selbsterfahrung als fortschreitende Effeminierung darstellt, stimmen schon die Naturalisten einen Tonfall markig-viriler Erneuerung an, den die Manifeste der frühen Avantgarde übernehmen. Dabei geht es jedoch nicht allein um iatsächliche Geschlechterkonflikte. Die Männlichkeitsrhetorik zielt vielmehr darauf ab, einer mit der Moderne heraufgekommenen Differenzierungskrise durch eine neue Kultur der Willensstörke und Entschlußkraft 'Herr' $z$ u werden. 
Nichts ist schneller in die Jahre gekommen als die Anfänge der Moderne. Was Deutschland betrifft, so erlebt sie eine ihrer Urszenen in einem bürgerlichen Salon. Ein Herrenabend um 1890. Der gastgebende Historiker führt das Wort. Er will seinen Gästen das „,moderne Ideal plastisch" machen. Thm schwebt dabei eine Frauengestalt vor Augen, doch stößt er auf energischen Protest

„Ein Weib? Also doch ein Weib!" unterbrach ungestüm der Moralist. „Und wozu dann der männliche Zug der Poesie, wozu der Kampf gegen das Backfisch- und Altjungferntum? O dieser Widerspruch in sich!“

„Durchaus keinen Widerspruch! durchaus ein Weib!“ fuhr der Historiker fort. ,Allerdings kein Backfisch und keine alte Jungfer, aber ein reifes Weib. Heissen die Frauen nicht noch immer „das schöne Geschlecht"? Sind sie nicht noch immer die gemütvoller Ausgebildeten, die Wächter des Edlen und Schönen, wo die rauhe Kraft des Mannes auf die materielle Seite des Lebens geht? Und ist die Göttin wahrer Poesie für jene Aftermuse mit Backfischnatur und Altjungfergelüsten verantwortlich, die eben der heuchlerischen Vertuschung des dahinsterbenden Geschlechts entsprach? -

Also ein Weib, ein modernes, d.h. vom modernen Geiste erfulltes Weib, zugleich Typus, d.h. ein arbeitendes Weib, d.h. von der materiellen Arbeit zum Dienste des Schönen und Edlen zurückkehrend, etwa auf dem Heimwege zu ihrem geliebten Kind, - denn sie ist keine Jungfrau voll blöder Unwissenheit über ihre Bestimmung, sie ist ein wissendes, aber reines Weib, und wild bewegt wie der Geist der Zeit, d.h. mit flatterndem Gewand und fliegendem Haar, mit vorwärtsschreitender Geberde, freilich nicht curch ihre überirdische Erhabenheit in den Staub nötigend, aber durch ihren Inbegriff aller irdischen Schönheit begeisternd mit fortreissend, - das ist unser neues Götterbild: die Moderne! (Wolff 1888/1971: 40)

Die Szene ist von dem Literaturkritiker Eugen Wolff entworfen. Wolff hat nicht im Sinn, die Berufstätigkeit deutscher Mütter zu heroisieren. Es geht in seinem Gemälde nicht um ein Rollenvorbild für Frauen. Nach Art der Nationaldenkmäler des 19. Jahrhunderts veranschaulicht die weibliche Allegorie das höhere Streben eines männlichen Kollektivs. Dynamisch in die Zukunft ausschreitend, soll die Göttin der Moderne ,materielle Arbeit" und Poesie miteinander vereinen und auf diesem Weg einen Kult der ,irdischen Schönheit" inaugurieren. Einer derart schwungvollen Vision können sich die versammelten Herren nicht widersetzen. Der anwesende Dichter weiht sich mit großer Geste „,zum Dienste der Moderne“, und selbst der über die Höhe solcher Gedankenflüge in Schweiß ge-ratene „Bürger", der dem Treffen der Intellektuellen bisher nur als Außenseiter beigewohnt hat, tritt in den Bund mit ein (Wolff 1888/1971: 40ff).

Bei aller Minnerhetorik ist der neue Kult der Salondisputanten, in den Wolff sein Plädoyer für die Erneuerung der Kunst einmünden läßt, eine Angelegenheit unter Männern und soll das auch bleiben. Die allegorische Szene arbeitet mit klaren geschlechtlichen Ausgrenzungen. Ein ,reifes Weib", ,, ein wissendes, aber reines Weib" steht dem Wortführer als Gottheit der Moderne vor Augen. Sie ist frei von „Backfischnatur und Altjungfergelüsten“, das heißt von vagierendem, herrenlosem Begehren. Diesen Tenor stimmen alle Proklamationen der Moderne im ausgehenden 19. Jahrhundert an. Das Manifest der Gruppe 'Durch', das 1887 in der Allgemeinen Deutschen Universitätszeitung erscheint, zieht aus dem neuen "Kunstideal" der Moderne, das die Antike-Verehrung verdrängen soll, gleich auch die erotischen Konsequenzen:

Bei solchen Grundsätzen erscheint ein Kampf geboten gegen die überlebte Epigonenklassizität, gegen das sich spreizende Raffinement und gegen den blaustrumpfartigen Dilettantismus. (zit. n. Wunberg 1971:2)

Oder mit den Worten des naturalistischen Programmatikers Kurt Pfütze--Grottewitz:

Ich denke mir den Zukunftsstil einfach, klar und mannhaft. Es wird kein Spielen sein mit pomphaft aufgeputzten Redensarten, kein schwülstiges Versteckenspielen mit gesuchten Ausdrücken und zerrissenen Sätzen, kein zimperliches, kleinliches Salonparlieren. Unumwunden, durchsichtig und markig wird die zukünftige Entwicklungsdichtung die grossen Gedanken und Ideale der neuen Ära sprachlich objektivieren. (Pfütze-Grottewitz 1891/1971: 83) 
Das sind bekannte Töne. So forciert innovativ sich die Kunstkritiker geben, so wenig originell ist ihre Diktion. Der Furor gegen Pomp, Raffinement, Koketterie, gegen französischen Salonstil und Frauenschriftstellerei, schließlich der Ruf nach einer viril-markigen Sprache waren in Deutschland schon einmal, gut hundert Jahre zuvor, im Schwange (Koschorke 1998). Nicht anders hatten nämlich die Stürmer und Dränger die Nationalisierung der deutschen Literatur eingeleitet. Im gleichen Stil, in dem Herder gegen die Entmannung der Sprache zu Feld zog, die er Gottsched und dem Rationalismus der Aufklärung anlastete (Herder 1877f.: I, 163), um dann prophetisch zu verkünden, man werde ,,in der Sprache zur alten Deutschen Einfalt und rauhen Stärke zurückkehren" (Herder 1877f.: II, 288), rechnet die naturalistische Generation mit der Hinterlassenschaft des 19. Jahrhunderts ab. Wieder scheint die Welt in die Hände von Schwächlingen und exaltierten Frauen geraten zu sein, und wieder steht eine Erneuerungsbewegung an - Erneuerung, das versteht sich, im Zeichen des Mannes.

Der kämpferische Ton hat den Modernen von 1890 nicht viel geholfen. Schon kurz nach der Jahrhundertwende erinnert man sich nur noch in Form von Nekrologen an sie. ${ }^{1}$ Schlimmer noch, dem Naturalismus wird nun seine bloß rezeptive, erleidende Einstellung gegenüber der Wirklichkeit vorgeworfen. „Er bedeutet", so befindet Hans Landsberg, „die Herrschaft des Weibes in der Literatur. Jede Schriftstellerin ist Naturalistin." (Landsberg 1904/1971: 173) Also muß die Moderne noch einmal anbrechen, diesmal in einer erklärtermaßen antinaturalistischen Wendung. Die neue Urszene spielt sich allerdings nicht in einem bürgerlichen Salon $a b$, sondern in Filippo Tommaso Marinettis Automobil. Genauer gesagt, in einem Abwasserkanal abseits der Straße, denn bekanntlich geht das Erste Manifest des Futurismus auf einen Verkehrsunfall zurück. Auch der Kult des Weiblichen der Moderne trägt nun andere Züge.

Oh, mütterlicher Graben, fast bis zum Rand mit schmutzigem Wasser gefüllt! Oh, schöner Abflußgraben einer Fabrik! Ich schlürfte gierig deinen stärkenden Schlamm, der mich an die heilige, schwarze Brust meiner sudanesischen Amme erinnerte... (Marinetti 1909/1993: 76)
2

Der männliche Körper hat im Industriezeitalter eine Serie von Demütigungen erfahren. Als Arbeitskörper, weil der zunehmende Einsatz von Maschinen traditionelle Konzepte von Körperkraft und deren gesellschaftlicher Wertschöpfung obsolet macht (cf. Rabinbach 1990). Als Kriegerkörper, weil ihn die Entwicklung der Waffentechnik, besonders der Artillerie, die Unzulänglichkeit seiner biologischen Ausstattung auf dramatische Weise fühlen läßt. Als Geschlechtskörper, weil die posttraditionale Gesellschaft nicht mehr auf Mythologien des Zeugungsvermögens und der Fruchtbarkeit gründet und dem Sexus infolgedessen eine untergeordnete Funktionsstelle zuweist. Und schließlich überhaupt als selbstbeherrsch. ter, orientierungsfähiger Organismus, weil die ungewohnten Angebote und Anforderungen der modernen Lebenswelt eine Fülle von nervlichen Funktionsstörungen nach sich ziehen.

Auf der Ebene der körperlichen Selbstwahrnehmung wird so die Dynamik der Modernisierung von einer scheinbar gegenläufigen, retroversen Bewegung begleitet: ständig findet sich die Anstrengung von vorzeitiger und übermäßiger Ermüdung, die Konzentration von nervöser Zerstreuung, das Begehren von Objektverlust, die Ausrichtung auf ein Ziel von einer depressiven Antriebslosigkeit bedroht (cf. Koschorke 1999). Fortschritt und Dekadenz, Energie und Entropie sind weder in der sozialen Semantik noch im Bereich subjektiver Befindlichkeit klar voneinander zu trennen (cf. Rabinbach 1990: 20ff, 47ff). Was speziell die Befindlichkeit des Mannes betrifft, so muß dieser das Vorwärtskommen der doch von ihm selbst in Gang gesetzten Moderne als zunehmende Schwächung, und das heißt nach den Einteilungen der bürgerlichen Geschlechteranthropologie: als Effeminierung erfahren (cf. Wagner 1991: 99ff). ${ }^{2}$

Alle diese Krisensymptome kulminieren im Krieg. Mit dem vielbeschworenen Heldentum in der Schlacht ist es nach den $\mathrm{Au}-$ gusttagen von 1914 bald vorbei. Vor dem Krieg hatte Valentine de Saint-Point in ihrem 'Manifest der futuristischen Frau' noch das Bild der wilden Heroengeliebten gezeichnet, die den Festzug der männlichen Kämpfer begleitet und ,immer den stärksten, den Sieger, belohnt" - eine dem antiken Rom nachempfundene Phantasie 
(Saint-Point 1912/1993: 94). ${ }^{3}$ Jahre später veranlaßt der Rückstrom der Kriegskrüppel von den Schlachtfeldern Marinetti dazu, den italienischen Frauen in einem flammenden Aufruf die ,gloriosi mutilati“ ans Herz zu legen, in deren Prothesenkörpern er die Utopie des futuristischen Maschinenmenschen aufscheinen sieht. ${ }^{4}$ Ein Appell, der aller Wahrscheinlichkeit nach bei seinem Zielpublikum nicht verfangen hat. Der Anblick verstümmelter Körper und zerschossener Gesichter stimuliert wohl die Montagetechnik der Dadaisten (Marcus 1992: 231), nicht aber den futuristischen Eros der Italienerinnen.

$\mathrm{Zu}$ den physischen Beschädigungen des Kriegerkörpers gesellt sich das Trauma der Traumatisierung durch den Krieg: die Erfahrung, daß immer mehr Soldaten, gegen Kriegsende sogar ganze Kompanieteile, dem Horror der Materialschlacht erliegen und $\mathrm{zu}$ Kriegszitterern, Kriegsneurotikern, zu Behandlungsfällen für die Frontpsychiatrie werden. Warum diese Demütigung so tiefgreifend ist - sie spielt, jedenfalls in Deutschland, eine kaum zu unterschätzende Rolle für die Psychobiographie des faschistischen Mannes -, wird erst klar, wenn man rekonstruiert, aus welchen Motiven der Kriegsausbruch eine so enthusiastische Zustimmung fand.

Die Apologeten des Krieges, zu denen anfangs mehr oder minder die gesamte deutsche Intelligenz zählt, sieht in dem militärischen Kräftemessen eine seit langem ersehnte Gelegenheit, radikal mit der Vergangenheit als ganzer zu brechen. In dieser Hinsicht be-steht eine unübersehbare Funktionsäquivalenz zwischen ästhetischer und militärischer Destruktion, Weltkrieg und Avantgarde. Die Liste der Hinfälligkeiten, von denen man sich mit einem Befreiungsschlag losmachen will, ist lang. Sie reicht vom emotionalen Treibhausklima der bürgerlichen Herkunftsfamilien über das wilhelministische Altherrenregime, gegen das sich seit Jahrhundertbeginn eine brüderschaftlich geprägte Jugendbewegung formierte, bis hin zum Komfort- und Sicherheitsdenken der liberalistischen Gesellschaft überhaupt. Dem kann ich hier nicht weiter nachgehen. Auf der Liste steht aber auch jene dunkle und verschlingende Erotik, in der das ausgehende 19. Jahrhundert geschwelgt hatte, und damit der gesamte Komplex der dekadenten Gefühlskultur. Und wieder stellt sich hier die Gedankenfigur einer überfälligen Rückgewinnung der Männlichkeit ein. ${ }^{5}$
Die soldatischen Männer ziehen, so paradox das klingen mag, gegen die Gefahr des Weiblichen zu Felde. „Dieser Krieg“, so schreibt der Kunsttheoretiker Wilhelm Worringer, ,ist über alle Gegensätze der Rassen und Nationen hinaus zu etwas viel Schlimmeren [sic] geworden: zu einer Art von Kampf zwischen zwei verschiedenen Geschlechtern." Und er fährt fort:

Ein Strindberg täte not, der mit all der bitteren Analyse des Wissenden hineinleuchte in die hoffnungslose Tragik dieses Geschlechterkampfs, den unsere Männlichkeit nun auszuleuchten hat mit der um keine phantastische Selbsttäuschung verlegenen Hysterie unserer Feinde.

Wir werden siegen in diesem Kampf; werden siegen mit den aufs höchste angespannten Muskeln unserer geistigen Energien, werden siegen nicht zuletzt auch mit der Gewalt unserer so glänzend organisierten und disziplinierten körperlichen Energien, mit jener Körperkraft, die man brutal nennen mag, die aber anbetungswürdig ist, wenn man es mit einer verzweifelten, unberechenbaren Schaden stiftenden Hysterie zu tun hat. (Worringer 1914/15:20)

Worringers Pamphlet ist nicht nur deshalb interessant, weil es den Krieg in einen geschlechterpolitischen Endkampf umdeutet - übrigens mit hörbaren Anklängen an die Rhetorik der Befreiungskriege, die auch ein Sich-Ermannen Deutschlands gegen das erotisch korrupte Frankreich feierten. ${ }^{6}$ Es nimmt darïberhinaus mit einer merkwürdigen Hellsichtigkeit das Psychodrama der Nachkriegsjahre vorweg. Nach Worringers Prophezeiung werden die soldatischen Männer zwar „äußerlich das Feld behaupten", aber zuletzt um ihren Triumph betrogen sein: „Denn das letzte Wort wird doch die Frau mit ihrer Hysterie behalten und was schlimmer ist, auch den letzten Applaus." (Worringer 1914/15: 20) Den heimkehrenden Kämpfern wird eine Phalanx aus Lüge und Hysterie in den Weg treten, an deren Spitze „die glänzend inszenierte und skrupellos bediente Weltpresse unserer Feinde" agiert (Worringer 1914/15: 21). Solchen Widersachern ist die schlichte Wahrheitsliebe der deutschen Kämp. fer nicht gewachsen.

So schlägt das Siegesversprechen in ein fatalistisches Szenario um, das schon in der Anfangsphase des Krieges wichtige Topoi des Nachkriegs-Revanchismus (Dolchstoßlegende, Lügenhaftigkeit der Weltpresse, innere Unbeugsamkeit des isolierten und verratenen 
Deutschland) sozusagen vorsorglich aufbereitet. Dieser Fatalismus bietet ein Indiz dafür, daß der Feind, anders als Worringer glauben machen will, nicht einfach draußen zu lokalisieren ist. Unter der Hand wird in dem kriegspropagandistischen Artikel aus der rein militärisch unterlegenen Weiblichkeit der Feinde das Syndrom einer femme fatale, die - wie Delila und all die anderen Heroinen der eigentlich überwunden geglaubten Kunst des fin de siècle - hinterrücks sogar den größten Helden bezwingt.

Worringer hat in dem expressionistischen Maler Franz Marc einen kritischen Leser gefunden. Marc, der den Krieg nicht aus nationaler, sondern aus europäischer Perspektive gutheißt, weil es ihm. um „Reinigung“, um die „keusche Majestät“ des „europäische[n] Gedanken [s]" und um den Durchbruch zum „Herrentum des Europäers" zu tun ist (Marc 1914/1978: 164), sieht auch die eigentliche Front nicht zwischen den kriegführenden Staaten verlaufen. Die Kriegspropaganda gehe fehl:

Denn in diesem Kriege kämpfen nicht, wie es in Zeitungen steht und wie die Herren Politiker sagen, die Zentralmächte gegen einen äußeren Feind, auch nicht eine Rasse gegen die andre, sondern dieser Großkrieg ist ein europäischer Bürgerkrieg, ein Krieg gegen den inneren, unsichtbaren Feind des europäischen Geistes. (Marc 1914/1978: 165)

Nach Maßgabe dieser Disposition stellt Marc Worringers Theorie des „'Geschlechterkampfes" " um. Der Krieg, so befindet er, ist kein

Kampf des männlichen Prinzips gegen das weibliche, unsres männlichen Rechtsgefühls gegen die weibliche Hysterie unsrer Gegner. [...]

Eher wäre es angemessen zu sagen, da $\beta$ Europa durch diesen blutigen Austrag der Waffen die eigne Hysterie überwinden, die giftigen und brüchigen Elemente, die dem alternden, in die Irre, Enge gegangenen Europa anhaften, ausstoßen will. [...] Der Europäer kämpft in diesem Kriege um seine Gesundung und Zukunft, mit Worringer zu reden, gegen die Hysterie und die alternden verkalkenden Elemente seines Leibes. (Marc 1914/1978: 167)

Die Soldaten, die sich an der Front gegenseitig beschießen, bekämpfen in Wahrheit gemeinsam einen gemeinsamen Feind. Dieser Feind ist niemand anderes als die Frau im Mann, das Weiblich-Hysteri- sche im Volkskörper Europas, der ein Blutbad nehmen muß, um sich zu regenerieren und dann „wie ein Mann“ (Marc 1914/1978: 166) aufzustehen. Umso verheerender die Wirkung, wenn der innere Feind den längeren Atem hat, wenn Katharsis und militärische Nervenkur fehlschlagen und im weiteren Verlauf des Krieges immer mehr Männer zu Hysterikern werden. Eine doppelte Niederlage, deren ideologische Konsequenzen unabsehbar sind. Der Stahlhelmkrieger, vom Grauen der Schlacht ausgebrannt und dadurch unerschütterlich geworden, gehört als Ikone des Nationalismus in der Weimarer Zeit ebenso dazu wie die antisemitische Umprägung jener "giftigen und brüchigen Elemente“, die den virilen Volkskörper zersetzen und deshalb 'ausgestoßen' werden müssen.

Im antimodernen Schrifttum von Max Nordau bis Hitler ist Moderne eine weibliches Syndrom. Aber mit solchen Ressentiments läuft immer ein Appell zur Ermannung und Erneuerung mit, der seinerseits modernistische, ja mitunter ausgeprägt avantgardistische Züge trägt. Die moderne Vermännlichungsrhetorik enthält zwei Leitmotive, die in enger Verbindung miteinander stehen. Es sind dies das Ideal der Klarheit und der Aufruf zu Willensstärke. Wenn der seit Ende des 19. Jahrhunderts anvisierte „Zukunftsstil" „, einfach, klar und mannhaft" sein soll (Grottewitz 1891/1971: 85), dann sind die sexualontologischen Ingredienzien dieser Seite der Modeme unübersehbar. Sie schlagen sich später auch in der Metaphy sik der Abstraktion nieder, von der die avantgardistischen Künstler beseelt sind. Es ist kein Zufall, daß derselbe Worringer, der den Weltkrieg als Kampagne zur Enthysterisierung begrüßt, mit seinem Buch 'Abstraktion und Einfühlung' von 1908 die erste Theorie der abstrakten Malerei verfaßt hat: eine Theorie, die den lebensfeindlichen „Abstraktionsdrang“ als Reaktion auf die sonst nicht zu bewältigende Kontingenz der Phänomenwelt rechtfertigt. ${ }^{7}$ Und Franz Marc, der Worringer in puncto Krieg noch überbietet, arbeitet in seinen kunstprogrammatischen Äußerungen mit einer Tiefensemantik, die Abstraktion, Geistigkeit, Transzendenz, Keuschheit und 
männliche Intellektualität als wenn nicht identische, so doch wesensverwandte Begriffsgrößen behandelt. ${ }^{8}$

In diese Begriffskette 1äßt sich bruchlos der Appell an die Steigerung des Willens einfügen. Auch der Wille ist nach dem Geschlechterdualismus ein maskulines Vermögen, und auch er profiliert sich vor dem Hintergrund einer allgemeinen Schwächung, Zersetzung und Anomie. Hier wie sonst ruht die avancierte Kunsttheorie auf semantischen Massenbewegungen auf; Übungen zur „Willensgymnastik“ oder "moralische[n] Orthopädie" (Gebhardt 1907: 2, 4) erfreuen sich um die Jahrhundertwende insgesamt großer Beliebtheit. Es sind Übungen, die der vielleicht schwerwiegendsten Demütigung, die dem männliche Körper im Modernisierungsprozeß des 19. Jahrhunderts auferlegt wird, entgegenarbeiten sollen: dem Faktum seiner immer bedrohlicheren nervlich-kognitiven Dekomposition.

Es würde hier zu weit führen, die Debatte über Nervosität um 1900 im Licht der damaligen Geschlechteranthropologie zu diskutieren. Man hat die in jener Zeit weitverbreiteten nervösen Phänomene mit allen nur möglichen Zumutungen der Moderne in Verbindung gebracht: mit der technischen Beschleunigung des Lebens ebenso wie mit der Reizüberflutung der anwachsenden Großstädte oder der sexuellen Repression (cf. Radkau 1998). Aber solche Teilerklärungen geben keinen hinreichenden Aufschluß darüber, warum auch für den Nervendiskurs die Rhetorik der Re-Virilisierung eine Art Generalnenner gebildet zu haben scheint. Klarheit, Entschiedenheit, Energie und Willen sind die männlich konnotierten Sčhlagworte, mit denen die zeitgenössischen Ratgeber versuchen, der nervösen Aufweichung der Ich-Konturen beizukommen.

Vielleicht sollte man derartige semantische Operationen gerade in ihrer Großräumigkeit beim Wort nehmen und weniger in inhaltlicher als in struktureller Perspektive befragen. Dann deuten all die Bemühungen, einer Effemination des männlichen Körpers entgegenzuwirken, nicht unmittelbar auf reale Geschlechterkonflikte, sondern auf eine allgemeine Krise der Unterscheidungen hin. ${ }^{9}$ Auf diese epistemologische Krise werden nachträglich Geschlechtskonnotationen appliziert, um ihr eine greifbare, leibhaftige Gestalt zu verleihen.

\section{Wie werde la Energilich?}

Bine vollftändige 2mleitung

sutr

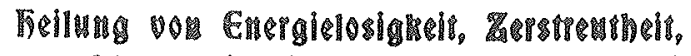

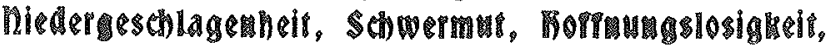
Mngstzus

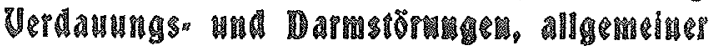

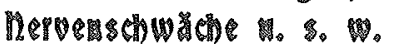

2lis manufript georubte Deroromungen.

Don

\section{Dr. Gevardi.}

7. prrmehrse und verbefferte 3uflage.

Seipzig

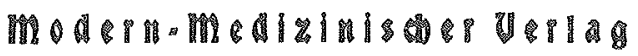

f. W. Bloterised

Abb. 6W. Gebhardt: „Wie werde ich Energisch?" (1907) 
Wenn die Nervosität im 19. Jahrhundert als eine Art von Entropie des Körperinneren aufgefaßt wird - und Entropie ist der physikalische Begriff für Entdifferenzierung ${ }^{10}-$, dann findet gleich ein doppelter Modelltransfer statt. Zunächst wird der zweite thermodynamische Hauptsatz seinem naturwissenschaftlichen Kontext entwendet, um eine gesellschaftliche Gesetzmäßigkeit auszudrücken: nämlich den unumkehrbaren Verlust an zielgerichteter sozialer Energie. Die Übernahme dieses weltanschaulich anverwandelten Entropie-Konzeptes in die Medizin wiederum ist als ein Indiz dafür lesbar, daß sich soziale Desintegrationsprozesse in bestimmten Symptomen einen körperlichen Ausdruck verschaffen. Und wenn sich nun solche Körperzustände in der zeitgenössischen Semantik als Einbruch in die männliche Verfaßtheit des Körpers darstellen, so deshalb, weil Kategorien wie Klarheit, Grenzziehung, Unterschiedenheit mit dem Prädikat 'männlich', Vermischung, Entgrenzung, Formlosigkeit dagegen mit dem Prädikat 'weiblich' versehen sind.

Die Rhetorik der Moderne beerbt und aktualisiert uralte Bestände der abendländischen Geschlechtermythologie. In dieser Mythologie ist das Differenzierende männlich, das Entdifferenzierende weiblich codiert. ${ }^{11}$ Umbruchphasen, in denen das gesellschaftliche Kategoriensystem nicht mehr nicht greift, Phasen der epistemologischen Unreinheit (cf. Douglas 1988, 1986) erscheinen unter solchen Vorzeichen fast notwendigerweise wie Perioden der Effemination. ${ }^{12}$ Als Chiffre für Entdifferenzierung ist das 'Weibliche' exakt an den Krisenpunkt der symbolischen Ordnung der Moderne gebannt - einer Ordnung, die wie jede andere auf der Gewährleistung von Differenzen beruht. Folgerichtig besteht ein Großteil der epistemischen Klärungsversuche jenes Epochenumbruchs darin, das 'Weibliche' aus dem Prozeß der Bedeutungsproduktion nach Möglichkeit auszuschließen, es als stumm, abwesend und wirkungslos zu behandeln (cf. Febel 1997).

Dieser erweiterte Ansatz schließt nicht aus, daß sich in die allgemeine Krise der Unterscheidungen konkrete und geschlechtlich spezifizierbare Krisenerfahrungen einschreiben. An solchen hat es gerade in der Zeit um 1900 nicht gemangelt. Doch ebensogut ist es möglich, daß Probleme, die ihrer Herkunft nach der Ebene der sozialen Semantik zugehören, in der Form eines pseudokonkreten Geschlechterkampfes ausagiert werden - weil eben die Geschlechter- differenz geeignete Metaphern für andere, abstraktere Differenzierungskrisen bereitstellt.

Die Ikonographie einer wiedergewonnenen Männlichkeit, die sich während der zwanziger und dreißiger Jahre unter so unterschiedlichen Parolen wie 'Neue Sachlichkeit' oder 'Triumph des Willens' ausbildet, legt diese zweite Lesart nahe. Denn ohne daß sich das empirische Verhältnis der Geschlechter zueinander fundamental gewandelt hätte, scheint sie die Forderungen der ästhetischen Vorkriegsprogramme weitgehend einzulösen. Sie modelliert einen zeitgemäßen Typus des entscheidungsfreudigen Mannes heraus, der sich mit der Technik und der Moderne ausgesöhnt und infolgedessen auch seine nervöse Antriebsschwäche abgelegt hat: den konstruktivistischen Architekten, neusachlichen Ingenieur, Planer, Flugpiloten, Journalisten oder Kameramann. Solchen Berufstypen, deren Liste sich noch ergänzen ließe, kommt offenbar eine inzwischen erfolgte kulturelle Akkommodation von Rationalisierungsleistungen zugute, an der die Avantgarden mit ihrer Bilderstürmerei und ihrer rigorosen Neuordnung der Phänomenwelt mitgewirkt haben. Sie profitieren überdies davon, daß zur gleichen Zeit auf breit gestreutem Niveau Entscheidungstheorien produziert werden, die, schlagwortartig verkürzt, auf einen inhaltsleeren und umso unbedenklicher handhabbaren Dezisionismus hinauslaufen. ${ }^{13}$

Irgendetwas ist - trotz aller faktischen Demütigungen - in der Schwellenzeit des Ersten Weltkrieges geschehen, das die diskursive Großwetterlage umpolt: vom Diskurs der Opfer der Modernisierung zu einem Diskurs der Teilhabe, der wiedererlangten Initiative. Das gilt sogar weitgehend unabhängig von den verschiedenen politischen Ausrichtungen. Jedenfalls wird allenthalben ein Generationenwechsel gefeiert, der einen radikalen Zeitenschnitt exekutiert. Nach dem Chaos setzen sich endlich Klarheit und Willenskraft durch. Man leidet nicht mehr unter der beschleunigten Zeiterfahrung der Moderne, sondern entdeckt ihre kinetische Energie. Überhaupt beginnt sich jetzt erst der schon um 1900 einsetzende Energiekult ${ }^{14}$ breit auszuwirken. Rationalisierung und Vitalismus, die beiden ehemals verfeindeten Prinzipien, gehen ein folgenträchtiges Zukunftsbündnis ein.

Das kann hier nur noch durch Stichworte belegt werden. Was die Literaturgeschichte angeht, so zeichnet sich ein signifikanter 
Stilwandel vom 'heißen' Apokalyptiker der expressionistischen Phase zum 'coolen' Überlebenskünstler im Dschungel der Großstadt ab. Durch ihre nüchterne, forciert unemotionale Diktion geben die neusachlichen Schriftsteller zu verstehen, daß sie einen Prozeß der Entsentimentalisierung erfolgreich durchlaufen haben und auf ihre Weise wieder sprachmächtig geworden sind. Im Idealtypus des Sportlers, den auch die Literaten verfechten - Brechts Adolatrie des Boxers ist das prägnanteste Beispiel dafür (Brecht 1995) -, sammeln sich alle positiven Gegenattribute der Dekadenzkritik. An der symbolischen Virilisierung des Körpers durch Sport sind nicht nur männliche Autoren beteiligt. Marieluise Fleißer schreibt in ihrem Essay über 'Sportgeist und Zeitkunst' von 1927:

Echter Sportgeist ist die aggressive Einstellung eines Menschen zu seinem eigenen Körper, wobei er an Hand bestimmter schwer zu erreichender Leistungen die Linie seines natïrlichen Körperwiderstandes durch seinen Willen zurückzudrängen versucht. [...] Das Resultat ist Entschlußfähigkeit. [...] Es ist Sache des Sportsmanns, in seinem Körper durch Energie dem Blitz möglichst weit entgegenzukommen. Sich herantreiben an die Nähe des Blitzes, Hochspannung in sich hervorrufen, ist jene Leidenschaft, die den treibenden Nerv des neuzeitlichen Menschentypus ausmacht. (Fleißer 1927/1994: 318f.)

Auch die Technikschriften der zwanziger Jahre atmen das Glück eines überstandenen Weltuntergangs. Franz Kollmanns Bildband 'Schönheit der Technik', 1928 in München erschienen, beeindruckt nicht nur durch fotografische Abbildungen von Kränen, Brücken, Industriebauten, Fahrzeugen und Maschinenteilen, sondern vermeldet nichts Geringeres als den Sieg der Form über die Degeneriertheit der alten, historistischen Bildung:

Für den erfürchteten Untergang Mitteleuropas sprach schon lange vor Ausbruch des Weltkrieges seine Formenarmut. Die schöne Gestalt, die bei den tausend Geräten täglichen Gebrauchs vorhanden sein soll, schien langsam auszusterben, bis plötzlich Erneuerung anhub. Aber sie kam nicht von dorther, von wo sie erwartet wurde, und kleinlicher Hader hinderte ihr Werden. Nicht aus klassisch geweihtem Boden erwuchs sie, sondern aus argwöhnisch beobachtetem Neuland. Die junge Bewegung aber hatte seelische Stoßkraft und überwand Widerstände. (Kollmann 1928: 9)
Eine radikale ästhetische Reform der Lebenswelt zeichnet sich ab und wird mit Konnotationen wie 'Entlastung' und 'Gesundung' verbunden. Vielleicht am deutlichsten artikuliert sich das Ethos der Totalen Erneuerung in den sozialhygienischen Begleitprogrammen zur avantgardistischen Architektur (cf. Koschorke 1999). „Dieser entschlossene Wille zu eigener, ursprünglicher, vom unbefangenen Erlebnis der Wirklichkeit befruchteter, von den Säften des Lebens durchbluteter Produktion ist der hervorstechendste Zug, der die Träger der modernen Stilbewegung geistig von dem Epigonen- und Asthetentum der akademischen Schule unterscheidet", schreibt Walter Curt Behrend 1928. „Aktive Söhne der neuen Zeit, nicht resignierende Enkel der Vergangenheit wollen sie sein." (Behrend 1928/1994: 143) - „Streben nach Klarheit", „strenge Vereinheitlichung", "Geist der Zusammengehörigkeit", "Ausdruck eines gemeinsamen Willens" lauten die entsprechenden Schlagworte bei Ludwig Hilberseimer, dem deutschen Pendant zu Le Corbusier (Hilberseimer 1927: 99). Die Krise scheint überwunden. Die siegreiche Avantgarde kehrt ihre herrische Seite hervor. 


\section{Anmerkungen}

1 „Und jetzt, wo wir an der Eingangspforte der modernen Dichtung zu stehen glauben, deren Entstehungszeit die Literaturgeschichte in das Ende der achtziger Jahre rückt, gerade jetzt werden wir von einem gelinden Schrekken erfasst, wenn wir diese Gründerjahre der neuen Poesie betrachten. Überall tote Namen und überlebte Werke, die nur eine wenig selbstbewusste, im naturalistischen Dogma aufgewachsene Wissenschaft mit peinlicher Treue registrieren konnte. Was sind uns heute die Holz und Schlaf, die Bleibtreu und Conradi, die Kirchbach und Walloth, die Scheerbart und Henckell, um aus der gewaltigen Totenliste nur einige einst hochberühmte Namen zu nennen?" (Landsberg 1904, in Wunberg 1971: 154)

${ }^{2}$, ,Zu sehr erinnert die ubiquitäre, fließende, unberechenbare Natur des Sexus den Inhaber bürgerlichen Besitzes an die 'zweite Natur', an die Willkür der ökonomisch-kapitalistischen Verhältnisse, denen er ausgesetzt ist. Einstige Realwerte haben sich verflüssigt, Vermögenswerte steigen und fallen mit den Bewegungen der Börse, seinem Einfluß entzogen, Gnade oder Ungnade bedeutend wie der Körper der Frau. In die Kontrolle und Eindämmung ihrer Sinnlichkeit flüchtet sich das männliche Kompensationsbedürfnis. [...] Der Nervöse dreht den Spieß seiner Schwäche, den gebrochenen Totempfahl seiner Männlichkeit um und greift an. Eine radikale Entwertung der Frau und alles dessen, was sie symbolisiert, ist der Kunstgriff männlichen Protestes."(Wagner 1991: 101f.)

${ }^{3}$ „Die Wollust ist eine Kraft, weil sie die Schwachen zermalmt, die Starken zur Hingabe von Kräften, also zu ihrer Erneuerung erregt. Jedes heroische Volk ist sinnlich. Die Frau ist der verlockendste Preis." (Saint-Point 1912, zit. n. Schmidt-Bergmann 1993: 94)

4 ,Accettiamo la collaborazione della guerra meccanica per colorare d'eroismo l'umanità scolorita dalla pace. Accendiamo le città quietiste e pacifiste colle linee violente e balzanti delle battaglie scolpite nel corpo umano.

La chirurgia, ha già iniziato la grande transformazione. Dopo Carrel la guerra chirurgica compie fulmineamente la rivoluzione fisioilogica. Fusione dell'acciaio e della Carne. Umanizzazione dell'acciaio, e metalizzazione della carne nell'uomo moltiplicato. Corpo motore dalle diverse parti intercambiabili e rimpiazzabili. Immortalità dell' uomo!..." (Marinetti 1916. Auf diesen Artikel hat mich Hanno Ehrlicher aufmerksam gemacht.)
${ }^{5}$ Hierzu und zum folgenden: Bischoff 1997. Bischoff befaßt sich mit der Art und Weise, wie die Geschlechterdifferenz bzw. 'das Weibliche' in die Kriegsthetorik Eingang finden. Hatte die Dekadenzliteratur ein Verwischen klarer Geschlechtergrenzen und eine Effeminierung des Mannes diagnostiziert, so wird nun der Krieg zur Bewältigung dieser Krise der Oppositionen herbeibeschworen." (65)

${ }^{6}$ Worringer selbst spricht vom „Geist von 1813“ (Worringer 1914/15: 21).

${ }^{7}$ Worringer 1908/1996: 48ff. Worringer unterscheidet seine Titelkategorien allerdings noch nicht historisch, sondern völkerpsychologisch. Er schreibt seinen Kommentar zur eben erst entstehenden abstrakten Malerei ex negativo, nämlich aus der Perspektive dessen, der noch vom ,Einfühlungsbedürfnis“ (47) herkommt. Jene Völker aber, die „der weiten, zusammenhanglosen, verwirrenden Welt der Erscheinungen" mit einer "geistigen Raumscheu" gegenübertraten (50), seien kunstpsychologisch ganz anders disponiert: „Ihr stärkster Drang war, das Objekt der Außenwelt gleichsam aus dem Naturzusammenhang, aus dem unendlichen Wechselspiel des Seins herauszureißen, es von allem, was Lebensabhängigkeit, d.i. Willkür an ihm war, zu reinigen, es notwendig und unverrückbar zu machen, es seinem absoluten Werte zu nähern. Wo ihnen das gelang, da empfanden sie jene Beglückung und Befriedigung, die uns die Schönheit der organischlebensvollen Form gewährt [...]." (51)

${ }^{8}$ Vgl. Marc 1914/1998. - Marc 1982. - Kandinsky/Marc 1911/1984. Eine genauere Analyse dieser Zusammenhänge ist von der entstehenden Habilitationsschrift von Claudia Öhlschläger zu erwarten.

${ }^{9}$ Dieser Begriff ist der Opfertheorie René Girards entlehnt, ohne daß deren Implikationen im folgenden mitverhandelt werden.

${ }^{10}$ „The obsession with fatigue in nineteenth-century thought was not merely a sign of the 'real' weariness of individuals in industrial society, but of the negative aspect of the body conceived as a thermodynamic machine capable of conserving and deploying energy. The body's fatigue was, as Helmholtz pointed out, a particular instance of entropy." (Rabinbach 1990: 47f)

"In wohlwollender Formulierung: „Gewiß ist es unrichtig, in dieser 'Undifferenziertheit' einfach ein Manko, ein Zurückgebliebensein zu sehen; vielmehr ist dies die durchaus positive, ein eigenes Ideal bildende Wesensart der Frau, die gleichberechtigt neben der 'Differenziertheit' des Mannes steht." (Simmel 1983: 94) 
${ }^{12}$ Ganz ähnlich hat die Aufklärung argumentiert und daraus den Auftrag abgeleitet, auch und gerade die Geschlechterordnung zu reformieren.

${ }^{13}$ Auf dem Niveau der (politischen) Philosophie: Krockow 1990. - Für Krockow wird der innere Zusammenhang zwischen den Autoren, denen seine Studie gewidmet ist, „,om Problem der Entscheidung her sichtbar. Jeweils ein Begriff ist in der Periode, in der die drei Denker zuerst hervortreten, von zentraler Bedeutung. Bei Jünger heißt er der Kampf, bei Schmitt die Entscheidung, bei Heidegger die Entschlossenheit. Eine gewisse Verwandtschaft ist sogleich deutlich, sie wird noch dadurch betont, daß die Begriffe, um 'sinnvoll' zu sein, materialen Gehalt, ein Wofür und Wogegen bzw. Wozu zu fordern scheinen, von allem materialen Gehalt aber gerade abgeschnitten werden. Kampf, Entscheidung und Entschlossenheit sollen rein als solche, gewissermaßen 'an sich' bedeutsam sein." (ebd.: 2)

Krockow deutet diese abstrakte Entschiedenheit als widersprüchliche Antwort auf den Verlust autoritativer Bindungen, wie er die Generation der Jugendbewegung geprägt hatte (34f).

${ }^{14}$ In Schriften wie Wilhelm Ostwalds 'Der energetische Imperativ', Leipzig 1912. - Vg1. zum Energetismus jener Zeit allgemein: Asendorf 1990.

\section{Literaturverzeichnis}

Asendorf, Christoph

1989 Ströme und Strahlen. Das langsame Verschwinden der Materie um 1900. Gießen.

Behrend, Walter Curt

1928/1994 „Vom Neuen Bauen“. In: Kristiana Hartmann (Hg.): trotzdem modern. Die wichtigsten Texte zur Architektur in Deutschland 1919-1933. Braunschweig, Wiesbaden, 1994: 137-145.

Bischoff, Doerte

1997 „Dieses auf die Spitze getriebene Mannestum'. Kriegsrhetorik und Autorschaft um 1914“. In: Kathrin Hoffmann-Curtius/Silke Wenk (Hg.): Mythen von Autorschaft und Weiblichkeit im 20. Jahrhundert. Marburg: 6072.

Brecht, Bertolt

1995

Der Kinnhaken und andere Box- und Sportgeschichten. Hg. Günter Berg. Frankfurt a.M.

Douglas, Mary

1986 Ritual, Tabu und Körpersymbolik. Sozialanthropologische Studien in Industriegesellschaft und Stammeskultur. Frankfurt a.M.

1988 Reinheit und Gefährdung. Eine Studie zu Vorstellungen von Verunreinigung und Tabu. Frankfurt a.M.

Febel, Gisela

1997

„'Poesie-Erreger' oder von der signifikanten Abwesenheit der Frau in den Manifesten der Avantgarde". In: Wolfgang Asholt/Walter Fähnders (Hg.): ,,Die ganze Welt ist eine Manifestation". Die europäische Avantgarde und ihre Manifeste. Darmstadt: 81-108.

Fleißer, Marieluise

1927/1994 „Sportgeist und Zeitkunst. Essay über den modernen Menschentyp". In: Marieluise Fleißer: Erzählende Prosa. Gesammelte Werke. Zweiter Band. Frankfurt a.M., 1994: 317-320. 
Gebhardt, W.

1907

Wie werde ich energisch? Eine vollständige Anleitung zur Heilung von Energielosigkeit, Zerstreutheit, Niedergeschlagenheit, Schwermut, Hoffnungslosigkeit, Angstzuständen, Gedächtnisschwäche, Schlaflosigkeit, Verdauungs- und Darmstörungen, allgemeiner Nervenschwäche u.s.w. 7. Aufl. Leipzig.

Grottewitz, Curt

1891/1971 „Neuer Stil und neue Schönheit“. In: Wunberg 1971: 8387.

Herder, Johann Gottfried

1877 ff Ueber die neuere Deutsche Litteratur. Eine Beilage zu den Briefen, die neueste Litteratur betreffend. In: Sämmtliche Werke. Hg. Bernhard Suphan. Bd. 1. Berlin.

$1877 \mathrm{ff} U$ Ueber Thomas Abbts Schriften. Der Torso von einem Denkmal, an seinem Grabe errichtet. In: Sämmtliche Werke, Bd. 2. Berlin: 249-294.

Hilberseimer, Ludwig

1927 Großstadtarchitektur. Stuttgart

Kandinsky, Wassily/Marc, Franz (Hg.)

1911/1984 Der Blaue Reiter [191//12]. Dokumentarische Neuausgabe. Hg. Klaus Lankheit. München.

Kollmann, Franz

1928 Schönheit der Technik. München.

Koschorke, Albrecht

1998 „Geschlechterpolitik und Zeichenökonomie. Zur Geschichte der deutschen Klassik vor ihrer Entstehung". In: Renate von Heydebrand (Hg.): Kanon Macht Kultur. Theoretische, historischen und soziale Aspekte ästhetischer Kanonbildungen. Stuttgart/Weimar: 581-599. hundert". In: Gerhart v. Graevenitz (Hg.): Konzepte de Moderne. DFG-Symposion 1997. Stuttgart/Weimar: 656 673. - Vorabdruck in: Leviathan. Zeitschrift für Sozialwissenschaft 27 (1999), Heft 1: 23-42.

Krockow, Christian Graf von

1990 Die Entscheidung. Eine Untersuchung über Ernst Jünger, Carl Schmitt, Martin Heidegger. 2. Aufl. Frankfurt a.M.NNew York

Landsberg, Hans

1904/1971 „Die moderne Literatur“. In: Wunberg 1971: 153-184.

Marc, Franz

1914/1978 „Das geheime Europa“. In: Klaus Lankheit (Hg.): Franz Marc. Schriften. Köln: 163-167.

1982 Briefe aus dem Feld. Hg. Klaus Iankheit/Uwe Steffen. München.

Marcus, Greil

1992 Lipstick Traces. Von Dada bis Punk - kulturelle Avantgarden und ihre Wege aus dem 20. Jahrhundert. Hamburg.

Marinetti, Filippo Tommaso

1909/1993 „Manifest des Futurismus“. In: Schmidt-Bergmann 1993: 75-80.

1916 „Donne, dovete preferiri i gloriosi mutilati“. In: L'Italia futurista, 1. Jg., Heft 2 (15. Juni).

Ostwald, Wilhelm

1912 Der energische Imperativ. Leipzig.

Rabinbach, Anson

1990 The Human Motor. Energy, Fatigue, and the Origins of Modernity. New York.

Radkau, Joachim

1998 Das Zeitalter der Nervosität. Deutschland zwischen Bismarck und Hitler. München, Wien.

Saint-Point, Valentine de

1912/1993 „Manifest der futuristischen Frau“. In: Schmidt-Bergmann 1993: 91-95.

Schmidt-Bergmann, Hansgeorg (Hg.)

1993 Futurismus. Geschichte, Asthetik, Dokumente. Reinbek. 
Simmel, Georg

1983

„Die Koketterie“. In: Georg Simmel: Philosophische Kultur. Über das Abenteuer, die Geschlechter und die Krise der Moderne. Gesammelte Essais. Berlin: 81-98.

Wagner, Nike

1991 „Zur Typologie des 'neurasthenischen' Mannes“. In: Nike Wagner u.a.: Mann, sei nicht so hysterisch. München: $97-$ 173.

\section{Wolff, Eugen}

1888/1971 „Die jüngste deutsche Literaturströmung und das Prinzip der Moderne“. In: Wunberg 1971: 3-42.

\section{Worringer, Wilhelm}

1908/1996 Abstraktion und Einfühlung. Ein Beitrag zur Stilpsychologie. Amsterdam.

1914/15 [Ohne Titel]. In: Zeit-Echo. Ein Kriegstagebuch für Künstler. München/Berlin, Heft 2: 20-22.

\section{Wunberg, Gotthard (Hg.)}

1971 Die literarische Moderne. Dokumente zum Selbstverständnis der Literatur um die Jahrhundertwende. Frankfurt a.M. 